\title{
Radio-Frequency Self-Referencing Technique With Enhanced Sensitivity for Coarse WDM Fiber Optic Intensity Sensors
}

\author{
Julio Montalvo, Member, IEEE, Orlando Frazão, Member, IEEE, José Luis Santos, \\ Carmen Vázquez, Senior Member, IEEE, and José Manuel Baptista, Senior Member, IEEE
}

\begin{abstract}
A self-referenced passive optical network (PON) with coarse wavelength division multiplexing (CWDM) is reported for remotely addressing optical intensity sensors with enhanced sensitivity. The self-referencing remote configuration is described as a finite-impulse-response (FIR) filter in reflective operation using two fiber Bragg gratings (FBG) and a fiber delay line. The antisymmetrical phase response of the configuration permits to achieve a self-referencing measurement parameter two times more sensitive than reported previously. To enhance the power budget of the network, CWDM devices with low insertion losses are used for spectral splitting of a radio-frequency (RF) modulated broadband light source (BLS). The network topology and the sensor's interrogation technique are theoretically analyzed and experimental results validating the models are reported.
\end{abstract}

Index Terms-Coarse wavelenght-division multiplexing (CWDM), digital filters, fiber Bragg gratings (FBG), fiber delay lines, optical intensity sensors, passive optical networks (PON), self-reference.

\section{INTRODUCTION}

$\mathbf{R}$ EMOTE addressing of photonic sensors using optical fibers and multiplexing schemes to measure the response of multiple sensing points have been a motive of research during the last years. Fibre optic intensity sensors, intrinsically safe and immune to electromagnetic interference (EMI), can be easily integrated in wavelenght-division multiplexing (WDM) networks and commercial devices and laboratory prototypes are available for the measurement of several parameters such

Manuscript received January 30, 2008; revised July 17, 2008. Current version published April 17, 2009. This work was supported by COST Action 299Optical Fibres for New Challenges Facing the Information Society "FIDES", Spanish CICYT TEC2006-13273-C03-03-MIC and FACTOTEM-CM S-0505/ ESP/000417, in part by Mobility Action 2007 (University Carlos III of Madrid), and in part by Integrated Action Luso-Española E-29-08.

J. Montalvo and C. Vázquez are with Universidad Carlos III de Madrid, Department de Tecnología Electrónica, 28911 Madrid, Spain (e-mail: julio.montalvo@uc3m.es; cvazquez@ing.uc3m.es).

O. Frazão is with INESC Porto, Unidade de Optoelectrónica e Sistemas Electrónicos, 4169-007 Porto, Portugal.

J. L. Santos is with INESC Porto, Unidade de Optoelectrónica e Sistemas Electrónicos, 4169-007 Porto, Portugal. He is also with Department de Física Universidade do Porto, 4169-007 Porto, Portugal (e-mail: josantos@fc.up.pt).

J. M. Baptista is with INESC Porto, Unidade de Optoelectrónica e Sistemas Electrónicos, 4169-007 Porto, Portugal. He is also with Department de Matemática e Engenharias, Universidade da Madeira, Campus da Penteada, 9000-390 Funchal, Portugal (e-mail: jmb@inescporto.pt).

Color versions of one or more of the figures in this paper are available online at http://ieeexplore.ieee.org.

Digital Object Identifier 10.1109/JLT.2008.2004949 as ultrasonics, temperature, pressure, humidity, corrosion and displacement [1]-[6]. Optical configurations based on fiber Bragg gratings (FBG) are effective approaches for addressing optical intensity sensors, because they provide reflective configurations that permit the use of a single fiber lead for both propagating directions of the light. Not only the use of FBG in optical sensor networks provide an effective and compact strategy for exploiting fiber links bidirectionally; actually, the sensitivity of the optical transducer can benhanced because it is possible to make the optical signal travel through the intensity sensor twice, once for each propagating direction of the light. Finally, FBGs are a well-known, low-cost and excellent technology to achieve WDM of optical sensors, because they can be used as spectral splitting devices of broadband light sources. Wavelength-division-multiplexed intensity sensor networks have been reported in reflective star and ladder topologies using FBGs and fused biconical wavelength selective couplers [7], [8] or CWDM devices [9]. Interrogation techniques for multipoint FBG sensors in series have been reported using modelocked wavelength-swept lasers [10] and Sagnac loop filters [11]. Recently, an ultralong-distance interrogation system based on FBGs has been demonstrated, allowing remote strain measurements at a distance of $120 \mathrm{~km}$ with no need for optical amplification [12].

FBG-based self-referencing configurations providing insensitivity to external power fluctuations, non-correlated to the sensor intensity modulation, have also been reported in reflective operation employing in the sensing points all-optical delay line filters [13], as well as Michelson and ring resonator configurations [14], [15]. Recently, we have reported a compact electro-optical design which avoids the need for fiber delay coils, thus achieving compact sensor heads, arbitrary modulation frequency and electronically reconfigurable operation in a single point [16].

In this paper, a reflective star network using CWDM devices and FBGs for multiplexing and interrogation of self-referencing optical fiber intensity sensors with enhanced sensitivity is reported. It is shown that the proposed multiplexing approach optimizes the power budget. The self-referenced measurement parameter is based on the optical configuration detailed in [13], but the Z-Transform technique is used for describing the new system with a doubled sensitivity, obtained by subtracting the output phase signals at two different modulation frequencies.

In Section II, the network architecture is presented and the power budget and scalability are studied. Section III contains 


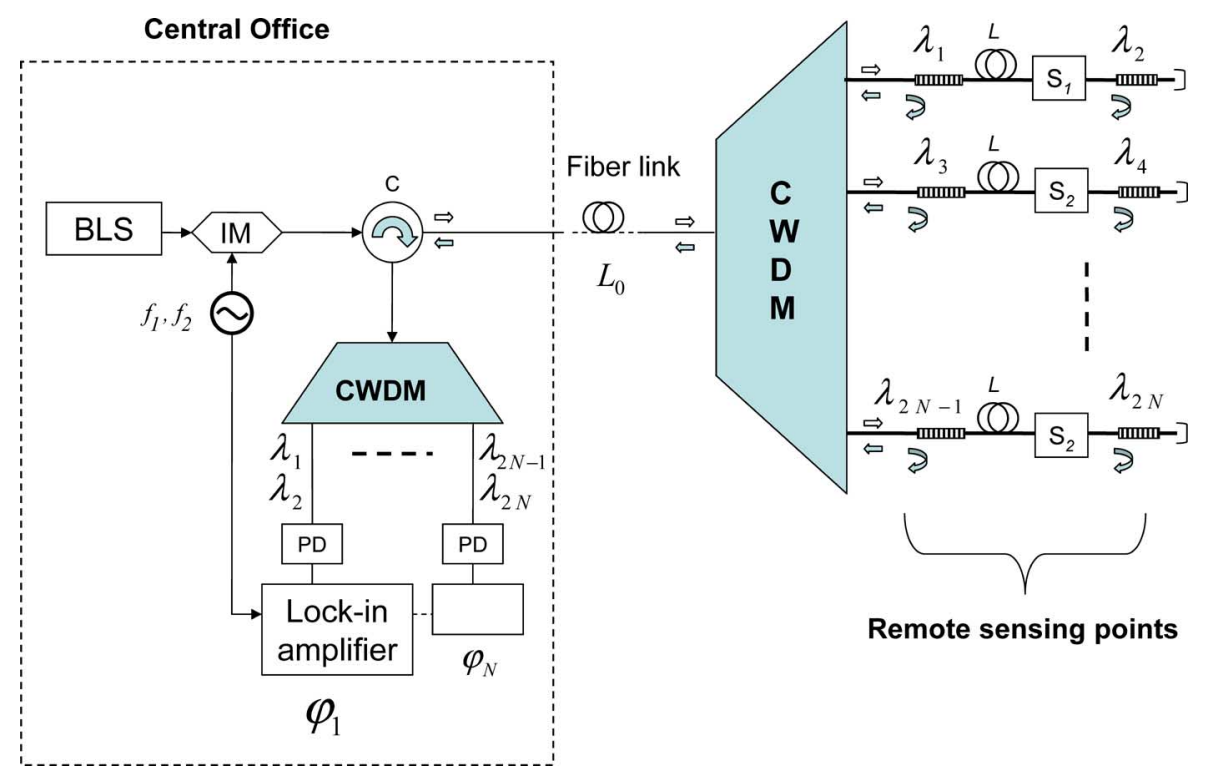

Fig. 1. Schematic of the proposed CWDM network for supporting $N$ self-referenced optical fiber intensity sensors $\left(S_{i}, i=1, \ldots N\right)$ with enhanced sensitivity. BLS: broadband light source, IM: intensity modulator, C: broadband circulator, PD: photodetector.

the transfer function analysis of the remote sensing configuration, using the digital filter theory and the new definition of the self-referenced measurement parameter with enhanced sensitivity. The performance of an experimental CWDM sensor network is reported in Section IV, validating the theoretical models and confirming the self-referenced measurements and the sensitivity enhancement. Finally, the main conclusions of the work are reported in Section V.

\section{NETWORK TOPOLOGY}

The general topology consists of a bidirectional CWDM passive optical network (CWDM PON) in double star topology for remote interrogation of $N$ optical intensity sensors (Fig. 1). A total number of $18 \mathrm{CWDM}$ channels are available in the complete wavelength range of 1271 to $1611 \mathrm{~nm}$ defined in ITU G.694.2, and a low-water-peak fiber (ITU G.652C-D) can be used for this wide spectrum of transmission.

With the proposed topology, the PON access to the sensors is achieved by means of a central office (CO) where the terminal equipment, both transmission and reception stages, are located. The distribution network consists of a primary fiber cable linking the $\mathrm{CO}$ to the remote sensing area. A secondary optical distribution network with low optical loss is deployed in this area by means of a CWDM bidirectional device with $N$ ports, achieving a remote quasi-distributed reflective sensor array $\left(S_{i}\right.$, $i=1, \ldots, N)$.

In the telecommunications environment, the WDM PON approach [17] has been recently developed by some industrials for broadband access aiming at 10-Gbit/s downstream networks, as a different solution to next generation $10 \mathrm{Gbit} / \mathrm{s}$ Ethernet PON (IEEE 802.3av) or 10-Gigabit PON (ITU-T G.984), which are based on power-splitting rather than in WDM. Communication WDM PONs have been recently featured for simultaneous provisioning of high-speed internet access, television and telephone (triple play service) [18], [19], even though standardized systems are not available yet. As the amount of information and the signal bandwidths are much higher than in sensor networks, arrayed-waveguide gratings (AWG) for dense WDM (DWDM) instead of CWDM devices are usually employed.

\section{A. Transmission Stage}

The optical transmitter consists of a radio-frequency (RF) intensity-modulated broadband light source (BLS). This BLS can be built either with an array of transmitters with central wavelengths $\lambda_{1}, \cdots, \lambda_{2 N}$ or with a single optical source with a wide spectrum, such as a super-luminiscent diode or erbium-doped fiber source, when operating in C-L band. Two different modulation frequencies $f_{1}, f_{2}$ excite the intensity modulator (IM) and immediately after the transmission stage, a broadband circulator is located in order to launch the downstream optical broadband signal into the PON. The modulated light reaches the remote sensing area through the primary fiber link.

\section{B. Reception Stage}

The broadband circulator receives the reflected multiplexed optical upstream with the sensor information. The upstream optical signal is demultiplexed by a CWDM device and delivered to an array of $N$ photodetectors (PD) and lock-in amplifiers operating as phase detectors, one for each sensor channel. If a single lock-in amplifier is available, either optical or electronic switching devices commercially available can be used to connect the amplifier to one of the $N$ signals, thus leading to a more compact and cost-effective reception stage.

\section{Sensor Configuration}

For each sensor channel $i$, two FBGs with central wavelengths $\lambda_{2 i-1}$ and $\lambda_{2 i}$ are emplaced in the remote sensing area. The generic wavelength $\lambda_{2 i-1}$ represents the reference channel for sensor $S_{i}$, while $\lambda_{2 i}$ is the sensor wavelength, because it reflects the optical signal containing the sensor-induced power modulation $H_{i}$ depending on the measurand (see Fig. 1). A symmetric bidirectional intensity sensor $S_{i}$ and an identical 
(a)

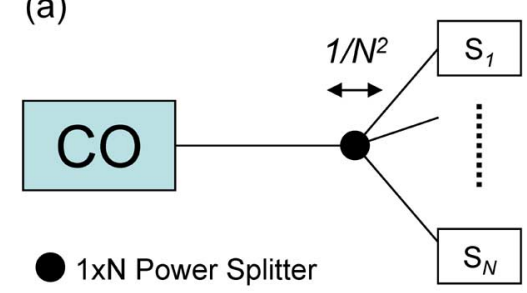

(b)

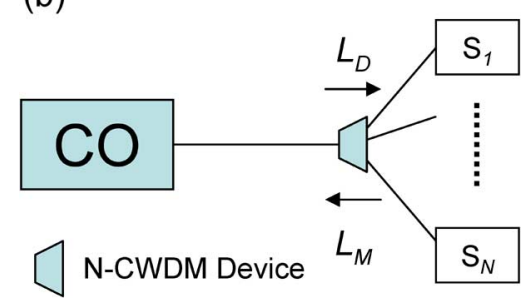

Fig. 2. Schematics of passive optical sensor networks using (a) power splitter and the (b) coarse MUX/DEMUX in the distribution stage.

fiber delay coil with length $L$ is located between the two FBGs at each sensing point. The self-reference mechanism provided by this reflective configuration and its performance are studied in Section III.

\section{Analysis of Power Budget}

By using CWDM devices in the sensor area, instead of the traditional broadcast power splitting approach, see Fig. 2, a power budget enhancement for the generic pair of wavelengths $\lambda_{2 i-1}$, $\lambda_{2 i}$, corresponding to sensor $S_{i}$, is achieved. Table I shows the symbols and definition of all the network parameters involved in the power attenuation perceived by the $\mathrm{CO}$ between the transmission and the reception stage.

The power attenuation of each short-reach WDM point-topoint connection established in the secondary fiber distribution section is considered negligible with regards to the attenuation of the main fiber link.

The total power attenuation $B_{R}(\mathrm{i}), B_{S}(\mathrm{i})$ for a generic reference and sensor wavelength $\lambda_{2 i-1}, \lambda_{2 i}$, respectively, have the expressions

$$
\begin{aligned}
& B_{R}(i)=L_{\mathrm{CU}} \cdot L_{\mathrm{CD}} \cdot 10^{-\frac{2 \alpha}{10} L_{0}} \cdot L_{D}^{2} \cdot L_{M} \cdot R_{R i} \\
& B_{S}(i)=L_{\mathrm{CU}} \cdot L_{\mathrm{CD}} \cdot 10^{-\frac{2 \alpha}{10} L_{0}} \cdot L_{D}^{2} \cdot L_{M} \cdot T_{i}^{2} \cdot R_{S i} \cdot H_{i}^{2} .
\end{aligned}
$$

The value of the attenuation induced in the distribution stages corresponding to the power splitting and CWDM approaches for $N$ sensors are shown in (3) and (4), respectively

$$
\begin{aligned}
A_{P S} & =1 / N^{2} \\
A_{\mathrm{CWDM}} & =L_{D} \cdot L_{M} .
\end{aligned}
$$

The generic power attenuation $B_{R}(\mathrm{i})$ versus the length of the main fiber is shown in Fig. 3 for different number of sensors $N$. In this figure, the advantages of the CWDM approach are clearly illustrated, as the power attenuation is much lower for any fixed

\begin{tabular}{|c|c|}
\hline Symbol & Definition \\
\hline$\overline{R_{R i}}$ & Reflectivity of the reference FBG for channel $i$ \\
\hline$T_{i}$ & $\begin{array}{l}\text { Power loss induced by the fibre-delay coil and the } \\
\text { reference FBG at sensor channel wavelength } \lambda_{2 j}\end{array}$ \\
\hline$\lambda_{2 i-1}$ & Central wavelength of the reference FBG for channel $i$ \\
\hline$R_{S i}$ & Reflectivity of the sensor FBG for channel $i$ \\
\hline$\lambda_{2 i}$ & Central wavelength of the sensor FBG for sensor $S i$ \\
\hline$\alpha$ & $\begin{array}{l}\text { Attenuation coefficient of optical fibre (typical value } \\
\text { for G.652 equals } 0.2 \mathrm{~dB} / \mathrm{km} @ 1550 \mathrm{~nm} \text { ). }\end{array}$ \\
\hline$L$ & Length of the fibre delay coil at each sensor point \\
\hline$L_{0}$ & Length of the main fibre link \\
\hline $10^{-\frac{\alpha}{10} \cdot 2 L_{0}}$ & Total power attenuation within the main fibre link \\
\hline$H_{i}$ & Power modulation ${ }^{\text {a }}$ induced by sensor $S_{i}$. \\
\hline$L_{C D}$ & $\begin{array}{l}\text { Insertion loss of the broadband circulator in the } \\
\text { downstream direction ( } \mathrm{CO} \rightarrow \text { Sensors })\end{array}$ \\
\hline$L_{C U}$ & $\begin{array}{l}\text { Insertion loss of the broadband circulator in the } \\
\text { upstream direction (Sensors } \rightarrow \mathrm{CO})\end{array}$ \\
\hline$L_{D}$ & $\begin{array}{l}\text { Insertion loss of the CWDM device operating as } \\
\text { optical demultiplexer }\end{array}$ \\
\hline$L_{M}$ & $\begin{array}{l}\text { Insertion loss of the CWDM device operating as } \\
\text { optical multiplexer }\end{array}$ \\
\hline
\end{tabular}
value of $N$ and $L_{0}$.
TABLE I

NETWORK PARAMETERS DEFINITION

a As the sensor wavelength $\lambda_{2 i}$ passes through sensor $S_{i}$ twice, the total sensor-induced power modulation is $H_{i}^{2}$.
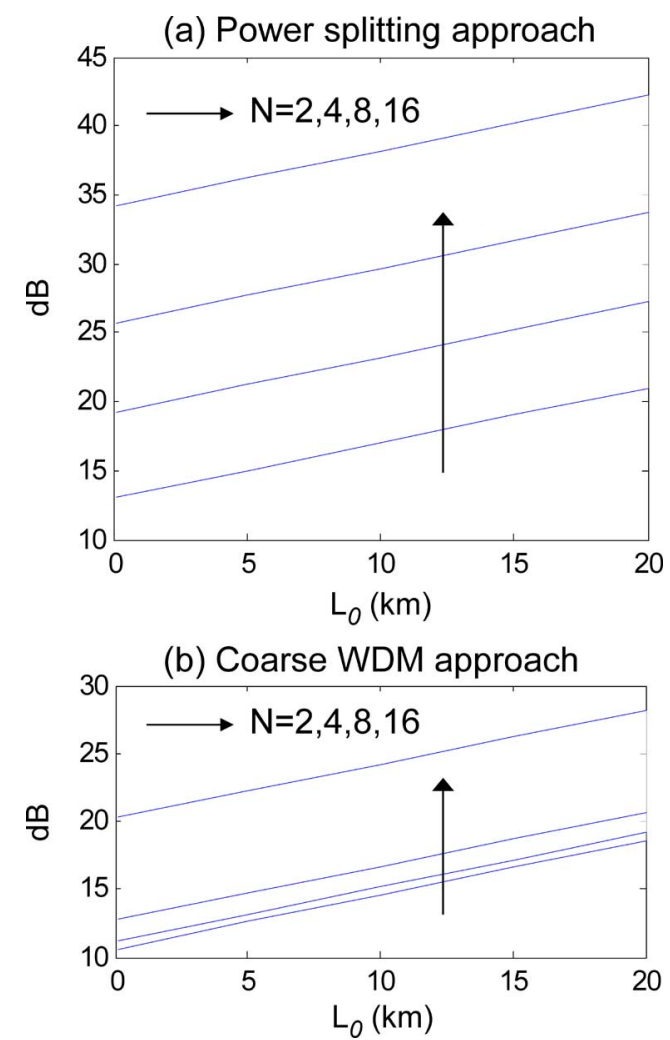

Fig. 3. Bidirectional insertion loss comparative at the reference wavelength, $B_{R}(i)$, between (a) the $1 / \mathrm{N}$ power splitting and (b) the N-CWDM approaches versus length of the main fiber, $L_{0}$, for different number of channels $N$ using (1). $\alpha=0.2 \mathrm{~dB} / \mathrm{km}, L_{\mathrm{CD}}=L_{\mathrm{CU}}=2.6 \mathrm{~dB}, L_{M}=L_{D}$ from Table II (maximum values), $R_{R}(i)=1$. 


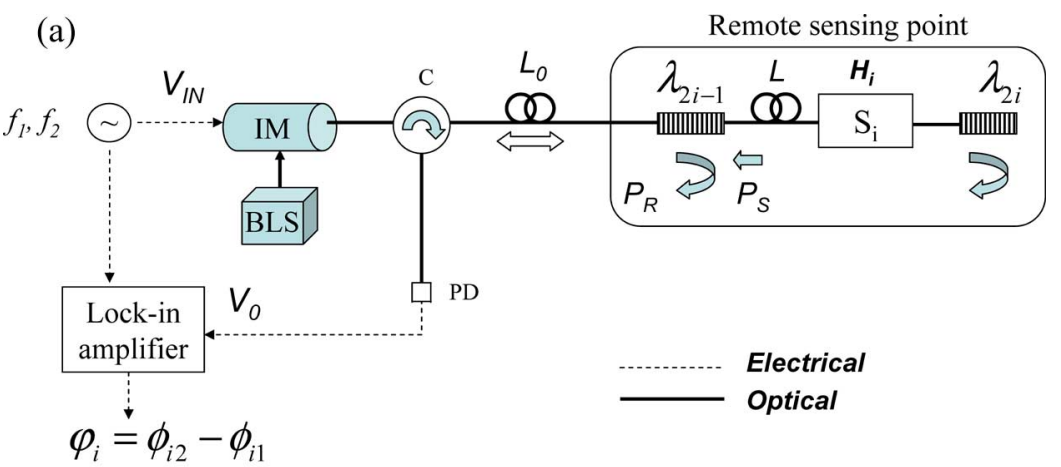

(b)

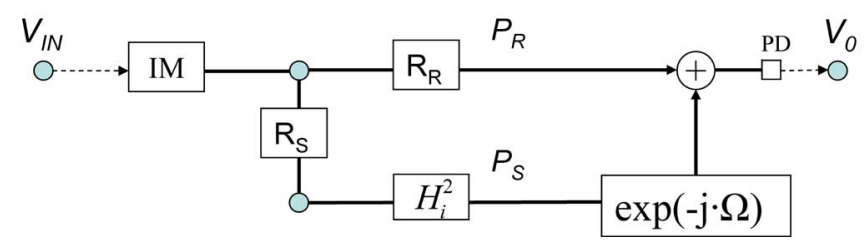

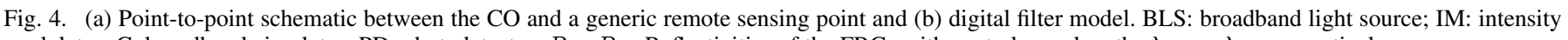
modulator; C: broadband circulator; PD: photodetector; $R_{R}, R_{S}$ : Reflectivities of the FBGs with central wavelengths $\lambda_{2 i-1}, \lambda_{2 i}$, respectively.

TABLE II

POWER ATTENUATION COMPARATIVE

\begin{tabular}{ccc}
\hline \hline $\begin{array}{c}\text { Number of } \\
\text { sensors }(N)\end{array}$ & Power splitting approach $^{\mathrm{b}}$ & Coarse WDM approach $^{\mathrm{c}}$ \\
\hline 2 & $6 \mathrm{~dB}$ & $2.8 \mathrm{~dB}(3.6 \mathrm{~dB}$ max. $)$ \\
4 & $12 \mathrm{~dB}$ & $3.2 \mathrm{~dB}(4.0 \mathrm{~dB}$ max $)$ \\
8 & $18 \mathrm{~dB}$ & $3.6 \mathrm{~dB}(5.0 \mathrm{~dB}$ max $)$ \\
16 & $24 \mathrm{~dB}$ & $8.6 \mathrm{~dB}(10 \mathrm{~dB}$ max $)$ \\
\hline \hline
\end{tabular}

b Values calculated using (3)

c Values calculated using (4) with values provided by manufacturer.

The main advantage of routing the optical power through a dedicated path for each pair of wavelengths, using CWDM devices, is that the optical power attenuation that takes place in the secondary distribution stage for $N$ sensors is much more significant using power splitting [see (3)] than using the CWDM approach [see (4)], see Table II.

\section{E. Scalability of the Network}

Commercially available Dense WDM multiplexers and demultiplexers, promoted by the high-speed optical communications market, can be used to scale the sensor network reported in this work. A DWDM upgrading of the 5 CWDM channels within the $\mathrm{C}+\mathrm{L}$ bands $(1528-1622 \mathrm{~nm})$ can be achieved by employing commercial bandsplitting filters based on athermal AWGs. These devices can achieve $50 \mathrm{GHz}$ channel separation and 40 channel counts within a single CWDM band. They do not require electrical power and operate over outdoor temperature range. On the other hand, apodized FBGs with central wavelength accuracy around 0.1 and $0.14 \mathrm{~nm}$ at $3 \mathrm{~dB}$ bandwidth are also technologically feasible [20], thus making possible to use the FBG-based self-referencing technique in a DWDM upgrading scenario. By adding 40 channels within each of the 5 CWDM bands in the 1520-1610 range, a maximum number over 200 multiplexed optical intensity sensors could be addressed with this DWDM upgrade approach. Nevertheless, high fabrication accuracy and stability of the FBGs are needed. As the power density per sensor channel is reduced, remote amplification or different transmission stages might also be required.

\section{NOVEL SELF-REFERENCED PARAMETER}

In this section, the response of the remote sensing configuration and the measurement technique realized in the $\mathrm{CO}$ are simultaneously considered for a generic sensor channel $i$.

The connection between the $\mathrm{CO}$ and a generic sensor $S_{i}$ can be modeled as a point-to-point bidirectional link at the corresponding waveband, which contains both corresponding wavelengths, $\lambda_{2 i-1}$ and $\lambda_{2 i}$ [Fig. 4(a)]. The self-referenced measurement parameter with enhanced sensitivity, namely $\varphi_{i}$, is defined and its performance versus sensor-induced power modulation and its frequency response are analyzed.

\section{A. Description of the Remote Interrogation Technique}

Two optical signals around the reference and sensor wavelengths, intensity-modulated at two frequencies $f_{k}(k=1,2)$ simultaneously, reach the remote sensing point. The intensity envelope of both optical signals can be described in the time domain using

$$
\begin{aligned}
& p_{r i}=P_{R i} \cdot\left(1+m_{r} \cdot \cos \left(2 \pi f_{k} \cdot t\right)\right) \\
& p_{s i}=P_{S i} \cdot\left(1+m_{s} \cdot \cos \left(2 \pi f_{k} \cdot t\right)\right)
\end{aligned}
$$

where $m_{r}, m_{s}$ are the modulation indexes of the reference and sensor channels at frequency $f_{k}$, respectively, being $P_{R i}, P_{S i}$ the average optical powers at the reference and sensor wavelengths, $\lambda_{2 i-1}, \lambda_{2 i}$.

Let $P_{R}, P_{S}$ be two complex numbers representing the reflected optical power of the intensity waves with frequency $f_{k}$ at the reference and sensor wavelengths, respectively.

The optical wave at the sensor wavelength $\lambda_{2 i}$ is phase-shifted $\Omega_{k}$ radians as a consequence of the group delay of the light in the fiber delay coil with length $L$. A power attenuation is also induced at this wavelength due to 
the intensity sensor transmission, $H_{i}$. The expressions of both complex phasors are

$$
\begin{aligned}
& P_{R}=m_{r} \cdot P_{R i} \cdot R_{R i} \\
& P_{S}=m_{s} \cdot P_{S i} \cdot R_{S i} \cdot T_{i}^{2} \cdot H_{i}^{2} \cdot \exp \left(-j \cdot \Omega_{k}\right) .
\end{aligned}
$$

The phase shift is obtained as follows:

$$
\Omega_{k}=\frac{2 \pi}{c} \cdot n_{g} \cdot f_{k} \cdot 2 L
$$

where $c, n_{g}$ are the light velocity in vacuum and the effective group index in optical fiber, respectively.

Once that both signals at the two wavelengths reach the CO, they add on an intensity basis in a linear photodetector, with responsivities $d_{r}, d_{s}$, at the reference and sensor wavelengths, respectively. These two parameters can be almost identical if the wavelengths of the two optical signals are very close.

The electric current signal obtained after the optical-to-electrical conversion in the photodetector can be described with the phasor

$$
I_{0}=d_{r} \cdot P_{R}+d_{s} \cdot P_{S}
$$

Finally, a current-to-voltage conversion with a global transimpendance constant $k_{0}$ takes place in the reception electronics and a proportional signal $V_{0}$ is obtained

$$
V_{0}=k_{0} \cdot I_{0}
$$

The sensor-induced power modulation, $H_{i}$, induces different phase variations of the output electrical signal, $V_{0}$, depending on the value of the modulation frequency $f_{k}$. In the next subsection, the digital filter model of the configuration is studied in order to analyze the frequency response using the Z-Transform and to obtain the novel self-referenced parameter with enhanced sensitivity, $\varphi_{i}$.

\section{B. Digital Filter Model}

Considering the transfer function in the electrical domain, being $V_{o}$ the electrical voltage proportional to the optical power at the receiver and $V_{I N}$ the electrical signal modulating the optical source at the input [Fig. 4(a)], and identifying a generic electrical phase-shift $\Omega_{k}$ with the characteristic transit time of an equivalent digital filter [21], [22], $z^{-1}=\exp \left(-j \cdot \Omega_{k}\right)$, the output response can be expressed in the Z-Transform domain as follows:

$$
\frac{V_{0}}{V_{I N}}=\alpha_{i} \cdot\left(1+\beta_{i} \cdot z^{-1}\right)
$$

being

$$
\begin{aligned}
\alpha_{i} & =k_{0} \cdot k_{r} \cdot d_{r} \cdot R_{R i} \\
\beta_{i} & =\frac{k_{s} \cdot R_{S i} \cdot d_{s}}{k_{r} \cdot R_{R i} \cdot d_{r}} \cdot T_{i}^{2} \cdot H_{i}^{2}
\end{aligned}
$$

where $k_{r}=P_{R i} \cdot m_{r} / V_{I N}, k_{s}=P_{S i} \cdot m_{s} / V_{I N}$ are the modulation efficiency constants at the reference and sensor wavelengths for channel $i$, respectively.

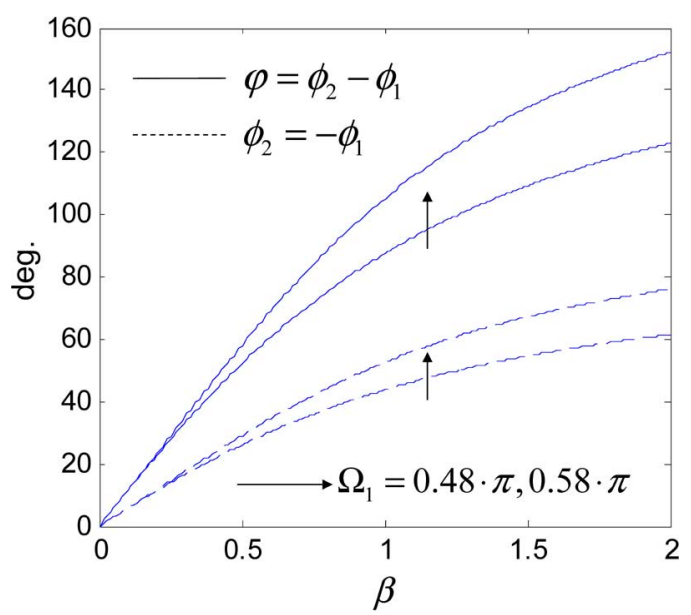

Fig. 5. Graphical representation of the novel self-referenced measurement parameter $\varphi$ (continuous lines) versus coefficient $\beta$ using two modulation frequencies $f_{1}, f_{2}$. The corresponding phase-shifting values are related by $\Omega_{2}=$ $2 \pi-\Omega_{1}$. The single-frequency parameters $\phi_{1}, \phi_{2}$, obtained at frequencies $f_{1}$, $f_{2}$, respectively, are drawn in dashed lines.

The expression of the output phase corresponding to sensor $i$ at angular frequency $\Omega_{k}$ is

$$
\phi_{i k}=\arctan \left(-\frac{\beta_{i} \cdot \sin \Omega_{k}}{1+\beta_{i} \cdot \cos \Omega_{k}}\right)
$$

For a fixed value of the modulation frequency, the parameter $\phi_{i k}$, namely single-frequency self-referencing parameter, depends only on the ratio-metric parameter $\beta_{i}$, which is insensitive to external power fluctuations that might take place in the optical path between the sensing point and the $\mathrm{CO}$, thus performing as a self-reference measurement parameter.

It is relevant to remark that $\phi_{i k}$ is antisymmetrical with respect to $\Omega_{k}=\pi$

$$
\phi_{i k}\left(\Omega_{k}\right)=-\phi_{i k}\left(2 \pi-\Omega_{k}\right) .
$$

This is a key issue for the definition of the novel self-referencing parameter with enhanced sensitivity, as it is shown in the next subsection.

\section{Definition of the Measurement Parameter}

By carefully choosing the values of the modulation frequencies $f_{1}, f_{2}$

$$
\Omega_{2}=2 \pi-\Omega_{1}
$$

the condition $\phi_{i 1}=-\phi_{i 2}$ is fulfilled, thus the novel self-referencing parameter $\varphi_{i}$, namely phase-difference self-referencing parameter, can be defined as follows:

$$
\varphi_{i}=\phi_{i 2}-\phi_{i 1}
$$

Because of the antisymmetry property of $\phi_{i k}, \varphi_{i}$ equals $2 \cdot \phi_{i 2}$ by definition, so the new self-referencing parameter using two modulation frequencies enhances two times the sensitivity of the single-frequency parameters $\phi_{i k}$ (Fig. 5). 


\section{MEASUREMENTS}

\section{A. Experimental setup}

A CWDM PON has been built using single-mode fiber in order to experimentally validate the phase-difference self-referencing parameter $\varphi$ simultaneously for two wavelength-multiplexed intensity sensors. The experimental setup follows the schematic shown in Fig. 1 for $N=4$.

Two bidirectional demultiplexers with four CWDM channels within the $\mathrm{C}$-band have been used at the remote sensing area and in the reception stage. These band-splitting devices show a flat shape with $0.5 \mathrm{~dB}$ bandwidths around $16 \mathrm{~nm}$ and low loss of $1.6 \mathrm{~dB}$. The two channels with central wavelengths $1530 \mathrm{~nm}$ (sensor $S_{1}$ ) and $1550 \mathrm{~nm}$ (sensor $S_{2}$ ) have been reserved for multiplexing two micro-displacement intensity sensors made of tapered single-mode fibers.

The reflective sensor configuration at each remote sensing is realized with one pair of FBGs for each channel. In total, 4 FBGs were used with a $3 \mathrm{~dB}$ bandwidth of approximately $0.25 \mathrm{~nm}$ and reflectivities around 50\%. Low-cost FBGs with reflectivities around $90 \%$ can be fabricated for improving the network power budget. The central wavelengths of the FBGs are $\lambda_{1}=$ $1533.3 \mathrm{~nm}, \lambda_{2}=1535.6 \mathrm{~nm}$ (sensor 1 ) and $\lambda_{3}=1550.9 \mathrm{~nm}$, $\lambda_{4}=1552.8 \mathrm{~nm}$ (sensor 2). Identical fiber coils with a length of $450 \mathrm{~m}$ and the corresponding intensity sensor were emplaced between the two FBGs at each sensing point. The fiber coils at all the sensing points must be identical in order to share the two modulation frequencies at the transmission stage for all the sensors; otherwise, the operation point of the measurement technique would be different for each sensor, which is not a desirable situation. In order to avoid this restriction, it is possible to replace the fiber delay coils with electrical filters at the reception stages [16], thus achieving a flexible and re-configurable operation point for each sensor, although an additional photodetector and optical demultiplexer are required for each sensor.

A super-luminiscent erbium-doped (SLED) fiber source with a broadband emission spectrum within the $C$-band and a total emitted power of $84 \mathrm{~mW}$ has been employed as BLS to address simultaneously the two intensity sensors in the CWDM network. This broadband light source has been intensity modulated by RF signals in the transmission stage with frequencies $f_{1}=75 \mathrm{kHz}$ and $f_{2}=150 \mathrm{kHz}$. For the fiber delay length of $450 \mathrm{~m}$, these two modulation frequencies provide angular frequencies $\Omega_{1}=0.65 \cdot \pi$ and $\Omega_{2}=1.35 \cdot \pi$ radians, with opposite phase responses $\left(\phi_{i 1}=-\phi_{i 2}\right)$ at each value of the power modulation coefficient $\beta_{i}$ and good linearity, thus allowing to achieve the self-referencing parameter with enhanced sensitivity, $\varphi_{i}$.

The two intensity sensors $S_{1}$ and $S_{2}$ are made of single-mode fiber tapers whose power transmission coefficients $H_{i}$ change due to the bending loss in single-mode fiber induced by microdisplacement. Fig. 6 shows the operation principle of the intensity sensors (a) and the calibration curve of $H_{1}$ in arbitrary units (a.u.) for sensor $S_{1}$ versus displacement (b). In order to test the hysteresis of the sensor, two sets of measurements, marked as forward and backward, were taken for increasing and decreasing values of the displacement, respectively.

A lock-in amplifier has been used in the reception stage in order to obtain the novel self-referencing parameters, $\varphi_{i}$ $(\mathrm{i}=1,2)$. For each sensor channel, a photodetector was located to obtain the corresponding electrical output signal, (a)
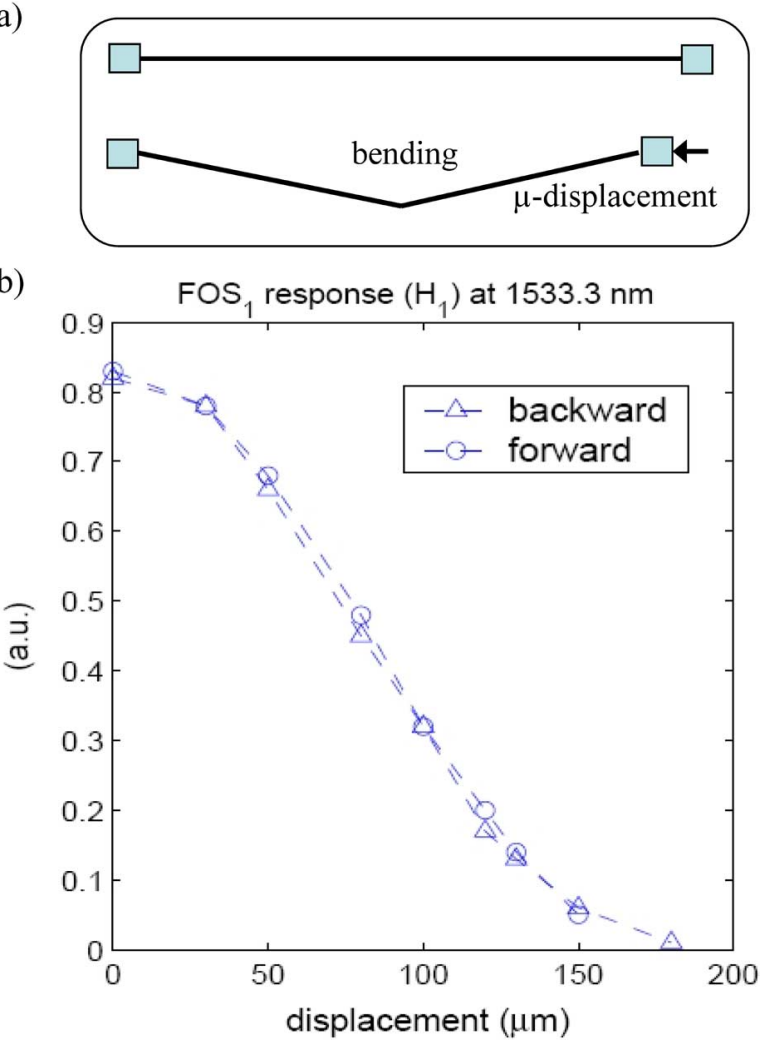

Fig. 6. (a) Sensor operation principle. (b) Calibration and hysteresis of the transmission coefficient $H_{1}$ for sensor $S_{1}(1530 \mathrm{~nm}$ band) versus displacement.

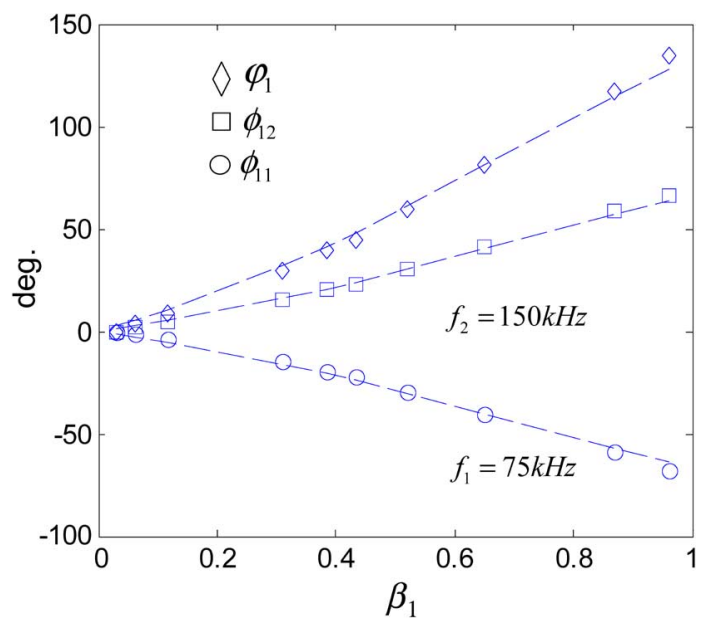

Fig. 7. Measurements of the self-referenced measurement parameters for sensor $S_{1}(1530 \mathrm{~nm}$ band). $L=450 \mathrm{~m}$.

thus measuring the single-frequency parameters $\phi_{i 1}, \phi_{i 2}$ by alternatively changing the value of the modulation frequency $f_{k}$. Finally, the phase-difference self-referencing parameter is calculated subtracting the two single-frequency parameters, $\varphi_{i}=\phi_{i 2}-\phi_{i 1}$.

Fig. 7 shows the measurements of the two single-frequency and the novel phase-difference self-referencing parameters for sensor $S_{1}$ versus the power modulation coefficient $\beta_{1}$. The theoretical fits are drawn in dashed lines, and a good agreement between theory and measurements is achieved. It has been estimated that $\beta_{1}=0.65 \cdot H_{1}^{2}$ and $\beta_{2}=0.74 \cdot H_{2}^{2}$. 


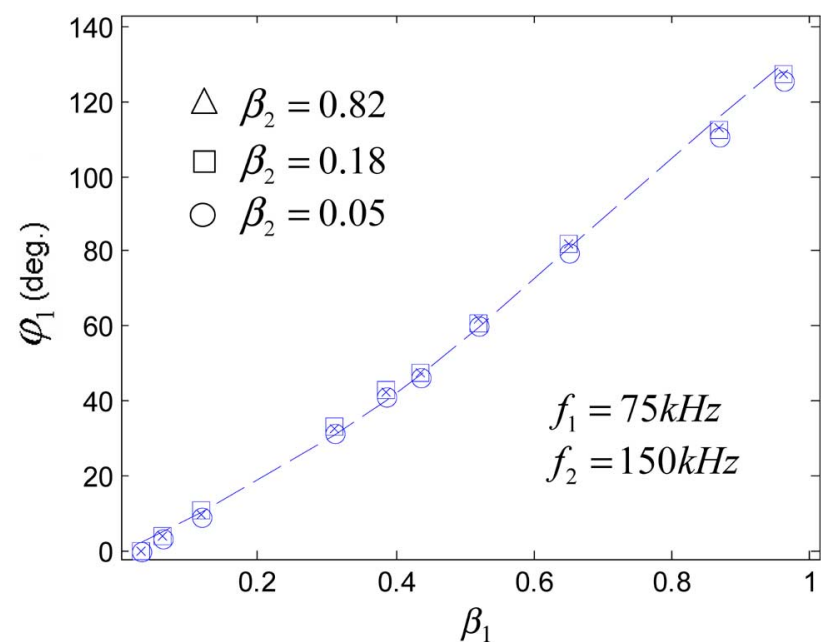

Fig. 8. Measurements of the phase-difference parameter $\varphi_{1}$ versus $\beta_{1}$ (sensor $S_{1}$ ). No crosstalk is induced due to changes in the power modulation coefficient $\beta_{2}$ of the adjacent channel (1550 nm band) for sensor $S_{2}$.

The performance of both phase-difference self-referencing parameters $\varphi_{1}, \varphi_{2}$ versus $\beta_{1}, \beta_{2}$, respectively, are exactly the same, as both sensor configurations share the modulation frequencies $f_{1}, f_{2}$ and the length of the fiber delay coils, $L$, see (9) and (15). The difference between the correction factors of $\beta_{i}$ for $\mathrm{i}=1,2$ are due to the different modulation efficiency constants $k_{r}, k_{s}$ of the intensity modulator and the different balance between the reflectivities of the reference $\left(R_{R i}\right)$ and the sensor $\left(R_{S i}\right)$ fiber Bragg gratings at the $1530 \mathrm{~nm}(\mathrm{i}=1)$ and $1550 \mathrm{~nm}$ $(\mathrm{i}=2)$ regions, see (14). For values of the ratio $R_{S i} / R_{R i}>1$, see (14), it is possible to increase the maximum values of $\beta_{i}$ and thus the measurement range of the phase-difference self-referencing parameter $\varphi_{i}$. This can be useful in order to compensate the bend and propagation loss at the fiber delay coil $T_{i}$, see Table I.

\section{B. Crosstalk Analysis}

In order to test the crosstalk between two sensors operating in adjacent CWDM channels, several measurements of the measurement parameter $\varphi_{1}$ (sensor $S_{1}$ ) were taken for different values of the power modulation $\beta_{2}$ (sensor $S_{2}$ ), as shown in Fig. 8. No correlation between the performance of $S_{1}$ and $S_{2}$ is noticed, so the two sensors can be interrogated simultaneously without mutual interference.

\section{Self-Reference Test}

Finally, the self-reference property of the sensitivity-enhanced measurement parameter $\varphi_{1}$ has been tested with regards to power fluctuations of the modulated BLS, using the same experimental setup of previous sections.

A single-mode variable optical attenuator (VOA) was located in the transmission stage between the BLS and the broadband circulator (Fig. 1), thus emulating unexpected power losses along the optical path linking the $\mathrm{CO}$ with the remote sensing area.

Fig. 9 shows that no correlation between the measurements of the self-referencing parameter $\varphi_{1}$ and the induced power attenuation up to $10 \mathrm{~dB}$ has been noticed. As the power attenuation

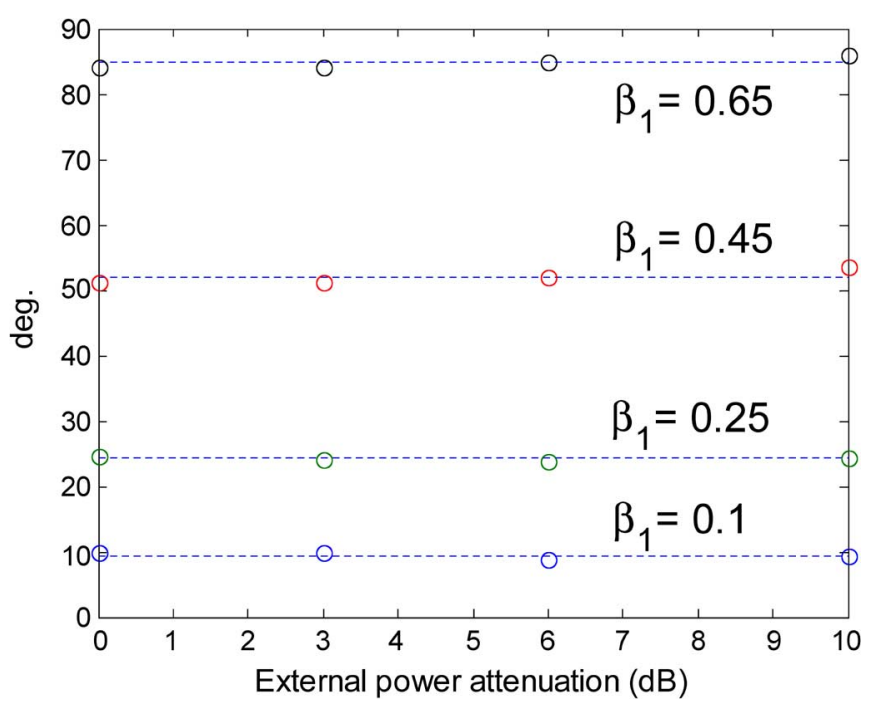

Fig. 9. Self-reference test of $\varphi_{1}$ versus power fluctuation up to $10 \mathrm{~dB}$ for different values of $\beta_{1}$.

induced by the VOA increased above $10 \mathrm{~dB}$, the phase measurements started to oscillate and the relative errors took unacceptable values. Hence, the maximum interrogation distance in the experiment is around $25 \mathrm{~km}$, which in a bidirectional link with a G.652 single-mode fiber (typ. attenuation equals $0.2 \mathrm{~dB} / \mathrm{km}$ ) induces $10 \mathrm{~dB}$ of attenuation, see (1) and (2).

\section{CONCLUSION}

A CWDM sensor network in reflective operation has been reported for self-referencing optical fiber intensity sensors with enhanced sensitivity. A novel self-referencing measurement parameter has been presented and experimentally validated, verifying a sensitivity two times higher with respect to previously reported results, at the expense of using an additional modulation frequency in the transmission stage. The Z-transform technique has been used for describing the system behaviour and, as in any reflective configuration, a single lead fiber is used in full-duplex operation. The reported measurement technique is suitable for quasi-static magnitudes whose time variations are in the order of the second or the minute, such as temperature, humidity, corrosion, gas concentration and pressure, among others.

A self-referenced CWDM network with two intensity sensors in adjacent channels at the 1530 and $1550 \mathrm{~nm}$ bands has been demonstrated with no crosstalk between sensors and tolerant to power fluctuations up to $90 \%$ of the available optical power. A maximum distance of $25 \mathrm{~km}$ between the $\mathrm{CO}$ and the remote sensing is estimated for a total emitted optical power of $84 \mathrm{~mW}$ in the $C-L$ band.

The reported network follows the topology of WDM PON for broadband access in the optical communications market, so lowcost off-the-shelf devices are available for building and scaling the network.

A DWDM upgrade of the network would allow to simultaneously address over 200 intensity sensors, but different optical sources and remote amplification might be required. 


\section{ACKNOWLEDGMENT}

The authors would like to thank P. Caldas for his help on the intensity sensors fabrication.

\section{REFERENCES}

[1] P. A. Lewin, C. Mu, S. Umchid, A. Daryoush, and M. El-Sherif, "Acousto-optic, point receiver hydrophone probe for operation up to $100 \mathrm{MHz}, "$ Ultrasonics, vol. 43, pp. 815-821, 2005.

[2] N. Díaz-Herrera, M. C. Navarrete, O. Esteban, and A. González-Cano, "A fiber-optic temperature sensor based on the deposition of a thermochromic material on an adiabatic taper," Meas. Sci. Technol., vol. 15, pp. 353-358, 2004.

[3] Y. Zhu and A. Wang, "Miniature fiber-optic pressure sensor," IEEE Photon. Technol. Lett., vol. 17, no. 2, pp. 447-449, Feb/ 2005.

[4] S. Dong, Y. Liao, and Q. Tian, "Intensity-based optical fiber sensor for monitoring corrosion of aluminium alloys," Appl. Opt., vol. 44, pp. $5773-5777,2005$.

[5] J. M. Corres, F. J. Arregui, and I. R. Matías, "Design of humidity sensors based on tapered optical fibers," J. Lightw. Technol., vol. 24, no. 11, pp. 4329-4336, Nov. 2006.

[6] J. M. Baptista, S. F. Santos, G. Rego, O. Frazão, and J. L. Santos, "Micro-displacement or bending measurement using a long-period fiber grating in a self-referenced fiber optic intensity sensor," Opt. Commun., vol. 260, pp. 8-11, 2006.

[7] S. Abad, F. M. Araújo, L. A. Ferreira, J. L. Santos, and M. López-Amo, "Multiplexing of fiber optic intensity sensors using fused biconical wavelength selective couplers," Electron. Lett., vol. 37, pp. 490-491, 2001.

[8] S. Abad, M. López-Amo, J. M. López-Higuera, D. Benito, A. Unanua, and E. Achaerandio, "Single and double distributed optical amplifier fiber bus networks with wavelength-division multiplexing for photonic sensors," Opt. Lett., vol. 24, pp. 805-807, 1999.

[9] J. Montalvo, C. Vázquez, and D. S. Montero, "CWDM self-referencing sensor network based on ring resonators in reflective configuration," Opt. Exp., vol. 14, pp. 4601-4610, 2006.

[10] X. Wan and H. F. Taylor, "Multiplexing of FBG sensors using modelocked wavelength-swept fiber laser," Electron. Lett., vol. 39, pp. 1512-1514, 2003.

[11] C.-S. Kim, T. H. Lee, Y. S. Yu, Y.-G. Han, S. B. Lee, and M. Y Jeong, "Multi-point interrogation of FBG sensors using cascaded flexible wavelength-division Sagnac loop filters," Opt. Exp., vol. 14, pp. 8546-8551, 2006.

[12] T. Saitoh, K. Nakamura, Y. Takahashi, H. Iida, Y. Iki, and K. Miyagi, "Ultra-long distance fiber Bragg grating sensor system," IEEE Photon. Technol. Lett., vol. 19, no. 20, pp. 1616-1618, Oct. 2007.

[13] S. Abad, M. López-Amo, F. M. Araújo, L. A. Ferreira, and J. L. Santos, "Fibre Bragg grating-based self-referencing technique for wavelengthmultiplexed intensity sensors," Opt. Lett., vol. 27, pp. 222-224, 2002.

[14] J. M. Baptista, S. Abad, G. M. Rego, L. A. Ferreira, F. M. Araújo, and J. L. Santos, "Wavelength multiplexing of frequency-based self-referenced fiber optic intensity sensors," Opt. Eng., vol. 43, pp. 702-707, 2004.

[15] C. Vázquez, J. Montalvo, D. S. Montero, and J. M. S. Pena, "Self-referencing fiber-optic intensity sensors using ring resonators and fiber Bragg gratings," IEEE Photon. Technol. Lett., vol. 18, no. 22, pp. 2374-2376, Nov. 2006.

[16] J. Montalvo, F. M. Araújo, L. A. Ferreira, C. Vázquez, and J. M. Baptista, "Electrical FIR filter with optical coefficients for self-referencing WDM intensity sensors," IEEE Photon. Technol. Lett., vol. 20, pp. $45-47,2008$

[17] A. Banerjee, Y. Park, F. Clarke, H. Song, S. Yang, G. Kramer, K. Kim, and B. Mukherjee, "Wavelength-division-multiplexed passive optical network (WDM-PON) technologies for broadband access: A review," J. Opt. Netw., vol. 4, pp. 737-758, 2005.

[18] S.-J. Park, C.-H. Lee, K.-T. Jeong, H.-J. Park, J.-G. Ahn, and K.-H. Song, "Fibre-to-the-home services based on wavelength-division-multiplexing passive optical network," J. Lightw. Technol., vol. 22, no. 11, pp. 2582-2591, Nov. 2004.

[19] D. J. Shin et al., "Low-cost WDM-PON with colorless bidirectional transceivers," J. Lightw. Technol., vol. 24, no. 1, pp. 158-165, Jan. 2006.

[20] R. Romero, O. Frazão, F. Floreani, L. Zhang, P. V. S. Marques, and H. M. Salgado, "Multiplexers and demultiplexers based on fiber Bragg gratings and optical circulators for DWDM systems," in Proc. 6th IEEE Int. Conf. High Speed Netw. Multimedia Commun. (HSNMC'03), 2003, pp. $442-451$.
[21] M. C. Vazquez, R. Civera, M. Lopez-Amo, and M. A. Muriel, "Analysis of double-parallel amplified recirculating optical-delay lines," Appl. Opt., vol. 33, p. 1015, 1994.

[22] M. C. Vazquez, B. Vizoso, M. Lopez-Amo, and M. A. Muriel, "Single and double amplified recirculating delay lines as fiber-optic filters," Electron. Lett., vol. 28, no. 11, pp. 1017-1019, 1992.

Julio Montalvo was born in Madrid, Spain, on May 1979. He received the M.S degree in telecommunication engineering in January 2003 from the Technical University of Madrid (UPM), Spain.

As a member of the Displays and Photonics Applications Group at UC3M, his research work is involved in optic filters, devices and systems design and characterization for coarse and dense wavelength-division multiplexed (WDM) networks for sensors and broadband access. Currently, he is also working in the telecommunications industry on optical broadband networks.

Dr. Montalvo won the doctorate special prize of UC3M in 2009

Orlando Frazão graduated in physics engineering (optoelectronics and electronics) from the University of Aveiro, Aveiro, Portugal. He is currently working towards the Ph.D. degree in physics at the University of Porto, Porto, Portugal.

From 1997 to 1998, he was with the Institute of Telecommunications, Aveiro, Portugal. Presently, he is a Researcher at Optoelectronics and Electronic Systems Unit, INESC Porto. He has published about 250 papers, mainly in international journals and conference proceedings, and his present research interests included optical fiber sensors and optical communications.

Mr. Frazão is a member of the Optical Society of America (OSA).

José Luis Santos graduated in applied physics and received the Ph.D. degree in physics from the University of Porto, Porto, Portugal, in 1983 and 1993, respectively.

Since 1987 he has been engaged on research and development of fiber optic sensors. He holds the position of Associate Professor of Physics Department of University of Porto, and he is also the manager of INESC Porto Optoelectronics and Electronics Systems Unit.

Dr. Santos is a member of OSA, SPIE and the Planetary Society.

Carmen Vázquez (M’99-SM’05) was born in Madrid, Spain, in May 1968. She received the M.S. degree in physics (electronics) in 1991 from Complutense University of Madrid, Spain, and the Ph.D. degree at Telecommunications Engineering School, Polytechnic University of Madrid (UPM), Madrid, Spain, in 1995.

She was a Fellow at TELECOM (Denmark) and worked in III-V integrated optics devices at "Telefónica Investigación y Desarrollo." In 1995, she joined Carlos III University of Madrid, Madrid, where she is currently working as an Associate Professor, Leader of the Photonics Applications Group, Vice-chancellor and was Head of the Electronics Technology Department for three years. Her current work includes optical signal processing, ring resonators, plastic optical fibers and LC, broadband access networks, RoF systems, filters, switches, fiber sensors design and sensor networks. She has published more than 100 papers in journals and conferences

Dr. Vázquez won the extraordinary doctorate prize of UPM in 1995 and biannual prize for young professor at UC3M in 2004. She participates as leader and researcher in Spanish and European Research Projects in Photonics. She is a member of SPIE, OSA, and SEO.

José Manuel Baptista graduated in electrical and computer engineering from the University of Porto, Porto, Portugal, in 1991. He received the M.Sc. degree in physics of laser communications from the University of Essex, Colchester, U.K. in 1992, and the Ph.D. degree in electrical and computer engineering from the University of Porto, Porto, Portugal, in 2002.

Currently, he is Assistant Professor of the Mathematics and Engineering Department of University of Madeira, Funchal, Portugal, and he is a Researcher of the Optoelectronics and Electronics Systems Unit of INESC Porto. His research interests are in the areas of fiber optic sensors and optical communications. 